Original Article

\title{
Can blood pressure be measured during exercise with an automated sphygmomanometer based on an oscillometric method?
}

\author{
Tomoyuki Shinohara, PT, PhD ${ }^{1 *}$, Naoko Tsuchida, PT, MS ${ }^{2)}$, Kanako Seki, $\mathrm{PT}^{2)}$, \\ Tomohiro Otani, PT, $\mathrm{MS}^{2)}$, Tatsuya Yamane, $\mathrm{PT}^{2)}$, Yamato Ishihara, $\mathrm{PT}^{2}$, \\ Chinatsu Usuda, $\mathrm{OT}^{2}$ ) \\ 1) Department of Physical Therapy, Faculty of Health Care, Takasaki University of Health and \\ Welfare: 501 Nakaorui-machi, Takasaki-shi, Gunma 370-0033, Japan \\ 2) Rehabilitation Center, Hidaka Hospital, Japan
}

\begin{abstract}
Purpose] This study investigated the reliability of an automated sphygmomanometer based on an oscillometric method, when used during exercise. [Subjects and Methods] Ten healthy subjects were included. Blood pressure (BP) was measured with an automated sphygmomanometer based on a cuff-oscillometric method. The experiment consisted of five tests: sitting posture at rest, walking with swinging the upper limbs, walking without swinging the upper limbs, walking on a treadmill, and riding a bicycle ergometer. Right and left brachial artery BP was measured twice at the same times. If the difference in systolic BP on bilateral testing was less than $15 \mathrm{mmHg}$, it was judged to be accurate, and accurate measurement rates were calculated. [Results] BP could not be measured in most limbs on walking with swinging the upper limbs, walking without swinging the upper limbs, or walking on a treadmill. The accurate measurement rates in bilateral limbs were $95.0 \%$ in sitting posture at rest, $0.0 \%$ on walking with swinging upper limbs, $5.0 \%$ on walking without swinging upper limbs, $15.0 \%$ on walking on a treadmill, and $65.0 \%$ on riding a bicycle ergometer. [Conclusion] An automated sphygmomanometer based on an oscillometric method was useful for BP measurement only at rest.

Key words: Blood pressure, Automated sphygmomanometer, Oscillometric method
\end{abstract}

(This article was submitted Jan. 28, 2017, and was accepted Mar. 11, 2017)

\section{INTRODUCTION}

To exercise safely in physical therapy, the exercise program should be modified according to an individual's habitual physical activity, physical function, and exercise responses ${ }^{1)}$. Blood pressure (BP) is useful for frequent monitoring of a subject's responses. For example, the stronger the exercise, the more easily the BP will rise. Moreover, systolic BP (SBP) rises during exercise more easily in the elderly than in the young. Various studies have reported how to exercise safely. Measurement of BP before, during, and after exercise found no significant differences; therefore, automatic rapid pedaling exercise was found to be safe even for frail older adults ${ }^{2}$. With muscle-strengthening exercise, BP was significantly higher during straight leg rising in the supine position than during knee extension in sitting position ${ }^{3)}$. BP is affected by exercise. Therefore, BP is measured to assess the safety and efficacy of exercise.

In general, measurers surround the upper arm with a mercury sphygmomanometer cuff to measure BP. Then, they listen for Korotkoff sounds at the brachial artery with a stethoscope. However, it is difficult to measure BP during exercise, because body movement often prevents detection of Korotkoff sounds. Precise measurement requires invasive BP monitoring, which can be measured sensitively in real time, using the brachial artery. BP is easy to monitor when the subject is resting, but is

\footnotetext{
*Corresponding author. Tomoyuki Shinohara (E-mail: tomo-e.pull.5312@ninus.ocn.ne.jp)

(C)2017 The Society of Physical Therapy Science. Published by IPEC Inc.

This is an open-access article distributed under the terms of the Creative Commons Attribution Non-Commercial No Derivatives (by-nc-nd) License $<$ https://creativecommons.org/licenses/by-nc-nd/4.0/>.
} 
difficult to measure during exercise and walking.

An automated oscillometric device added to a mercury sphygmomanometer may be useful for blood pressure measurement ${ }^{4}$. Recently, the automated sphygmomanometer has replaced the non-automated sphygmomanometer in hospitals and medical facilities. The automated sphygmomanometer uses oscillometric methods. Oscillometric methods measure BP by sensing the strength of the pressure in the cuff over the artery, and gradually releasing air. High reliability of oscillometric methods has been reported ${ }^{5-7)}$. An automated sphygmomanometer using oscillometric methods is an accurate, safe, and easy noninvasive method for monitoring BP, and is used in physical therapy. However, the main disadvantage is that such recorders do not work well during physical activity when there may be considerable movement artifact ${ }^{8}$. And oscillometric methods is very sensitive to movements due to the bandwidth of the signals, so the arm must be immobile ${ }^{9)}$. But, we experienced that BP could be measured while riding a bicycle ergometer and grasping a bar and there is not the experiment report that blood pressure was measured by oscillometric methods during exercise. It may be useful to measure BP during exercise without the arm mobile.

This study aimed to determine the reliability of an automated sphygmomanometer during exercise, for use by physical therapists.

\section{SUBJECTS AND METHODS}

We examined 10 healthy subjects (6 male; 4 female, mean age: $29.1 \pm 3.9$ years, range: $23-37$ years), with no history of illness that could affect autonomic nerve function, such as diabetes mellitus or hypertension. Subjects were provided verbal and written information about the study and written consent was obtained prior to inclusion.

Brachial artery BP was measured using an automatic cuff-oscillometric device (ES-H55D; Terumo Corporation). Our experiment consisted of five BP tests (Fig. 1): in sitting posture at rest, during walking at comfortable speed with swinging the upper limbs, during walking at comfortable speed without swinging the upper limbs, during walking on a treadmill at $3 \mathrm{~km} /$ hour while grasping a bar, while riding a bicycle ergometer and grasping a bar. The bilateral upper arms were fitted with cuffs, and after 20 to 30 seconds later right and left arm BP was successively measured twice at the same times. The cuff was pressurized to $200 \mathrm{mmHg}$. The order of the test was decided at random by lots. Breaks were permitted between tests.

We added the number of limbs in which we were able to measure BP and calculated the measurement rate every five tests. We calculated differences in SBP between bilateral limbs. If the difference in SBP was less than $15 \mathrm{mmHg}$, we considered the measurement to be accurate. A difference in SBP of 10 or $15 \mathrm{mmHg}$ or more between arms might help to identify patients who need further vascular assessment, and a difference of $15 \mathrm{mmHg}$ or more could be a useful indicator of risk of vascular disease or death ${ }^{10)}$.

All procedures were approved by the ethics committee of Hidaka Hospital (reference number 69), and the study followed all guidelines of the Declaration of Helsinki. We have no financial or personal relationships to disclose.

\section{RESULTS}

Mean SBP/diastolic BP ( \pm standard deviation) in sitting posture at rest was $116.8 / 66.2( \pm 10.3 / 14.7)$ on the right limb, and $120.5 / 66.4( \pm 8.7 / 14.1)$ on the left limb.

The measurement rates were $100.0 \%$ in sitting posture at rest, $40.0 \%$ on walking with swinging upper limbs, $57.5 \%$ on walking without swinging upper limbs, $42.5 \%$ on treadmill walking, and $97.5 \%$ on riding a bicycle ergometer (Table 1). BP

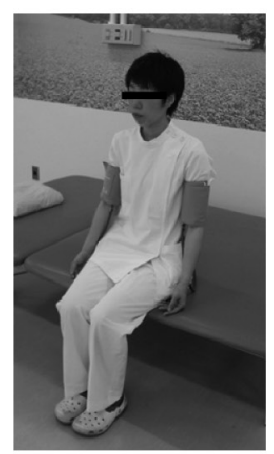

Sitting posture at rest

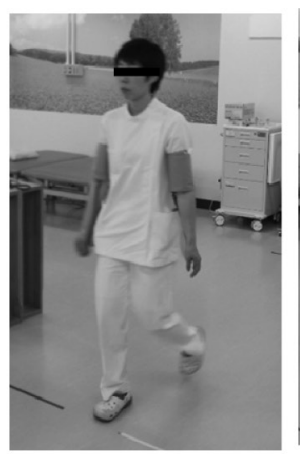

Walking with swinging the upper limbs

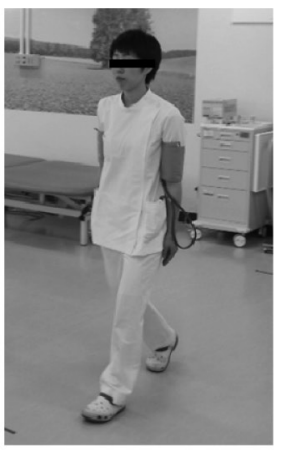

Walking without swinging the upper limbs

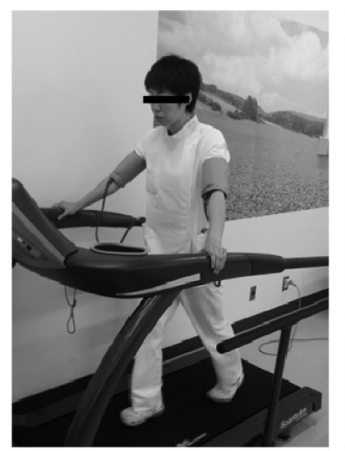

Walking on a treadmill

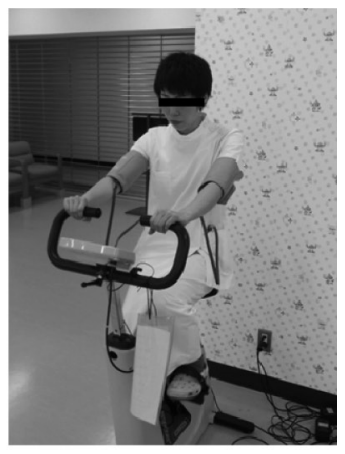

Riding

a bicycle ergometer

Fig. 1. BP measured in five tests 
could only be measured in all limbs in sitting posture at rest. On riding a bicycle ergometer, BP in only one limb could not be measured. We could not measure BP in most limbs on the three walking tests. Twenty measurable pairs were found in sitting posture at rest, with 4 pairs on walking with swinging upper limbs, 6 pairs on walking without swinging upper limbs, 8 pairs on walking on a treadmill, and 19 pairs on riding a bicycle ergometer (Table 2). Even when BP could be measured on both right and left sides, there was a difference of $15 \mathrm{mmHg}$ or more in many pairs (Table 3). The maximum difference was 82 $\mathrm{mmHg}$. A difference of $15 \mathrm{mmHg}$ or more was not considered accurate. Accurate measurement rates in bilateral limbs were $95.0 \%$ in sitting posture at rest, $0.0 \%$ on walking with swinging upper limbs, $5.0 \%$ on walking without swinging upper limbs, $15.0 \%$ on walking on a treadmill, and $65.0 \%$ on riding a bicycle ergometer (Table 2).

\section{DISCUSSION}

In this study, we investigated reliability of an automated sphygmomanometer based on an oscillometric method in five tests. BP could only be measured in sitting posture at rest. In another four tests, BP could not be measured in all limbs. When the difference in bilateral SBP was less than $15 \mathrm{mmHg}$ of BP that could be measured accurately, only sitting posture at rest showed a high accurate measurement rate $(95 \%)$. The next highest accurate measurement rate was on riding a bicycle ergometer, but the accurate rate was only $65 \%$. On walking with swinging upper limbs, walking without swinging upper limbs, and walking on a treadmill, the accurate measurement rates were low. Therefore, it is not useful to measure BP at the brachial artery with an automated sphygmomanometer based on an oscillometric method when subjects are walking. It is necessary

Table 1. The measurement rate in five tests

\begin{tabular}{lccc}
\hline & Measurable (limbs) & Unmeasurable (limbs) & Measurement rate \\
\hline Sitting posture at rest & 40 & 0 & $100.0 \%$ \\
Walking with swinging the upper limbs & 16 & 24 & $40.0 \%$ \\
Walking without swinging the upper limbs & 23 & 17 & $57.5 \%$ \\
Walking on a treadmill & 17 & 23 & $42.5 \%$ \\
Riding a bicycle ergometer & 39 & 0 & $97.5 \%$ \\
\hline
\end{tabular}

Table 2. The accurate measurement rate in five tests

\begin{tabular}{lccc}
\hline & Measurable (pairs) & Accurate measurement (pairs) & Accurate measurement rate \\
\hline Sitting posture at rest & 20 & 19 & $95.0 \%$ \\
Walking with swinging the upper limbs & 4 & 0 & $0.0 \%$ \\
Walking without swinging the upper limbs & 6 & 1 & $5.0 \%$ \\
Walking on a treadmill & 8 & 3 & $15.0 \%$ \\
Riding a bicycle ergometer & 19 & 13 & $65.0 \%$ \\
\hline
\end{tabular}

Table 3. Differences in SBP between bilateral limbs $(\mathrm{mmHg})$

\begin{tabular}{|c|c|c|c|c|c|c|c|c|c|c|}
\hline \multirow[t]{2}{*}{ Subject No. } & \multicolumn{2}{|c|}{$\begin{array}{c}\text { Sitting posture } \\
\text { at rest }\end{array}$} & \multicolumn{2}{|c|}{$\begin{array}{l}\text { Walking } \\
\text { with swinging } \\
\text { the upper limbs }\end{array}$} & \multicolumn{2}{|c|}{$\begin{array}{l}\text { Walking } \\
\text { without swinging } \\
\text { the upper limbs }\end{array}$} & \multicolumn{2}{|c|}{$\begin{array}{c}\text { Walking } \\
\text { on a treadmill }\end{array}$} & \multicolumn{2}{|c|}{$\begin{array}{c}\text { Riding a bicycle } \\
\text { ergometer }\end{array}$} \\
\hline & $1 \mathrm{st}$ & 2nd & $1 \mathrm{st}$ & 2nd & $1 \mathrm{st}$ & $2 \mathrm{nd}$ & $1 \mathrm{st}$ & 2nd & $1 \mathrm{st}$ & 2nd \\
\hline 1 & 12 & 2 & - & - & - & - & - & 55 & 0 & 16 \\
\hline 2 & 8 & 3 & 19 & - & 28 & 32 & 15 & 13 & 49 & 5 \\
\hline 3 & 5 & 8 & - & - & 16 & - & - & 14 & 32 & 10 \\
\hline 4 & 10 & 7 & - & - & - & - & 35 & 8 & 39 & - \\
\hline 5 & 7 & 14 & - & - & - & - & - & - & 7 & 16 \\
\hline 6 & 2 & 8 & - & - & - & - & - & - & 4 & 0 \\
\hline 7 & 9 & 9 & - & - & - & - & - & - & 7 & 15 \\
\hline 8 & 0 & 14 & 23 & - & - & 22 & - & - & 43 & 5 \\
\hline 9 & 5 & 14 & 82 & - & 14 & 42 & - & - & 4 & 5 \\
\hline 10 & 14 & 20 & 33 & - & - & - & 4 & 11 & 50 & 9 \\
\hline
\end{tabular}

-: BP could not be measured in either right and left 
to measure BP in sitting posture at rest. If we measure BP during use of a bicycle ergometer, we must assess the result of BP measured by the automated sphygmomanometer with caution. In nine of ten subjects, differences in SBP in bilateral limbs were less than $15 \mathrm{mmHg}$ on either the first or second measurement. This suggested that BP could be measured accurately if we measured twice. However, it is not practical to measure right and left brachial artery BP at the same time during exercise.

BP could not be measured in three walking tests. In the tests in which subjects walked with swinging upper limbs, we assume that vibration is significant. Therefore, we tried two tests without swinging upper limbs, i.e., simply walking and walking on a treadmill while grasping a bar. However, on these tests, the accurate measurement rates were very low. Even when subjects walked without swinging upper limbs, it was assumed that vibration interferes with BP measurement. Vibration results from pulse waves or motion during exercise; the two cannot be distinguished. We supposed that upper limb vibration on walking was influenced by motion of the lower limbs and trunk, and increased during walking in spite of arm placement. When subjects walked on a floor, vertical and horizontal sway of the center of mass occurred. From a low, central point in double limb support (for example, right initial contact), the center of mass moves upward and laterally (right mid-stance), drops to a second central low point (left initial contact), rises to a peak again (left mid-stance), and drops once more (second right initial contact). Each deviation is approximately $2 \mathrm{~cm}$ (up and to each side) ${ }^{11)}$. On the other hand, when subjects walked on a treadmill at $2.5 \mathrm{~km} / \mathrm{h}$ with both arms crossed, the trajectory of the center of mass in the frontal plane swayed transversely by approximately $3 \%$ of height ${ }^{12}$. If the subject's height is $160 \mathrm{~cm}, 3 \%$ is $4.8 \mathrm{~cm}$. In walking on both floor and treadmill, the trajectory of center of mass was more or less 4 to $5 \mathrm{~cm}$ in the frontal plane. Vibration on walking on the floor was the almost same as on walking on a treadmill.

There are several limitations in the present study. We used an automatic cuff-oscillometric device because it was small and easy to carry. Therefore, it was useful for measuring patient BP by a physical therapist. A device that can measure BP accurately during exercise is needed to verify the reliability of an automatic cuff-oscillometric device. It is also necessary to compare measurements between another device and an automatic cuff-oscillometric device. In this study, we compared BP with right and left measurements using automatic cuff-oscillometric devices. To measure BP during exercise accurately, it is necessary to distinguish pulse waves from the noise of motion during exercise. For example, a device that detects Korotkoff sounds tuned to the $\mathrm{R}$ wave on an electrocardiogram could measure $\mathrm{BP}$ during exercise. Korotkoff sounds tuned to the $\mathrm{R}$ wave can be distinguished from noise of motion.

In summary, the present study demonstrated the usefulness of an automated sphygmomanometer based on an oscillometric method. These are used widely by patients at home or medical workers. However, BP could only be measured accurately at rest, and could not be measured on walking and riding a bicycle ergometer. If it is necessary to measure BP and assess circulatory responses during exercise, we need to use the device to monitor BP during exercise other than an automatic cuff-oscillometric device.

\section{REFERENCES}

1) Garber CE, Blissmer B, Deschenes MR, et al. American College of Sports Medicine: American College of Sports Medicine position stand. Quantity and quality of exercise for developing and maintaining cardiorespiratory, musculoskeletal, and neuromotor fitness in apparently healthy adults: guidance for prescribing exercise. Med Sci Sports Exerc, 2011, 43: 1334-1359. [Medline] [CrossRef]

2) Sakuma H, Okuma O, Kobayashi M, et al.: Analysis of the reaction of cardiovascular system for frail older adults during the automatic rapid pedaling exercise. The Bulletin of Health Science University, 2010, 6: 135-141 (in Japanese).

3) Hasegawa T, Yamasaki H, Yamada S, et al.: Responses of heart beat and blood pressure during muscle strengthening exercise- the purpose of minimizing cardiac load-. Rigakuryohogaku, 1995, 22: 171-174 (in Japanese).

4) Elliott WJ, Young PE, DeVivo L, et al.: A comparison of two sphygmomanometers that may replace the traditional mercury column in the healthcare workplace. Blood Press Monit, 2007, 12: 23-28. [Medline] [CrossRef]

5) Yelderman M, Ream AK: Indirect measurement of mean blood pressure in the anesthetized patient. Anesthesiology, 1979, 50: 253-256. [Medline] [CrossRef]

6) Friesen RH, Lichtor JL: Indirect measurement of blood pressure in neonates and infants utilizing an automatic noninvasive oscillometric monitor. Anesth Analg, 1981, 60: 742-745. [Medline] [CrossRef]

7) Nystrom E, Reid KH, Bennett R, et al.: A comparison of two automated indirect arterial blood pressure meters: with recordings from a radial arterial catheter in anesthetized surgical patients. Anesthesiology, 1985, 62: 526-530. [Medline] [CrossRef]

8) Ogedegbe G, Pickering T: Principles and techniques of blood pressure measurement. Cardiol Clin, 2010, 28: 571-586. [Medline] [CrossRef]

9) Bonnafoux P: Auscultatory and oscillometric methods of ambulatory blood pressure monitoring, advantages and limits: a technical point of view. Blood Press Monit, 1996, 1: 181-185. [Medline]

10) Clark CE, Taylor RS, Shore AC, et al.: Association of a difference in systolic blood pressure between arms with vascular disease and mortality: a systematic review and meta-analysis. Lancet, 2012, 379: 905-914. [Medline] [CrossRef]

11) Perry J: Gait analysis: normal and pathological function. New Jersey: SLACK, 1992, pp 40-42.

12) Shinoda H, Sato H, Suzuki Y: Evaluation of gait stability based on variability of medio-lateral motion of the center of mass. Rigakuryoho Kagaku, 2008, 23: 55-60 (in Japanese). [CrossRef] 